\title{
ASTEROSEISMOLOGICAL PROBING OF THE THERMAL EVOLUTION OF WHITE DWARF STARS
}

\author{
G. FONTAINE and F. WESEMAEL \\ Département de Physique, Université de Montréal
}

\section{A Brief Review of the Properties of White Dwarfs}

It is generally believed that the immediate progenitors of most white dwarfs are nuclei of planetary nebulae, themselves the products of intermediate- and low-mass main sequence evolution. Stars that begin their lifes with masses less than about 7-8 $M_{\odot}$ (i.e., the vast majority of them) are expected to become white dwarfs. Among those which have already had the time to become white dwarfs since the formation of the Galaxy, a majority have burnt hydrogen and helium in their interiors. Consequently, most of the mass of a typical white dwarf is contained in a core made of the products of helium burning, mostly carbon and oxygen. The exact proportions of $\mathrm{C}$ and $\mathrm{O}$ are unknown because of uncertainties in the nuclear rates of helium burning.

It is a remarkable fact that isolated white dwarfs show a very narrow mass distribution, $M \sim 0.60 \mathrm{M}_{\odot}$, with a dispersion of $\sim 0.10 \mathrm{M}_{\odot}$. Also, it is currently believed that small traces of helium and hydrogen are left over after the mass loss phases have subsided. Because of the intense gravitational field characteristic of white dwarfs $(\log g \sim 8)$, elements rapidly segregate in the outer layers of such an object, with the lighter ones floating on top of the heavier ones. Hence, the expected structure of a typical newly-formed white dwarf is that of a compositionally-stratified object with a mass of $\sim 0.6 \mathrm{M}_{\odot}$ consisting of a $\mathrm{C} / \mathrm{O}$ core, surrounded by a thin, helium-rich envelope, itself surrounded by a hydrogen-rich layer; its average density is $\sim 10^{6} \mathrm{~g}$ $\mathrm{cm}^{-3}$. The respective thicknesses of the hydrogen and helium layers are not known a priori, and must depend on the details of pre-white dwarf evolution. On theoretical grounds, it is expected that the maximum amount of helium that can survive the hot planetary nebula phase is only $10^{-2}$ of the total mass of the star, while the maximum fractional amount of hydrogen is about $10^{-4}$. Recent observational results indicate, however, that the actual layer thicknesses in white dwarf stars may be many orders of magnitude smaller than these maximal values. Nevertheless, irrespective of their thickness, these layers remain extremely opaque, and regulate the energy outflow from the star. They consequently play an essential role in the evolution of a white dwarf.

\section{The Mechanical Structure and Thermal Evolution of White Dwarfs}

A dying star begins the final phase of its history in the form of an extremely hot, collapsed object which can only cool off: its nuclear energy sources are depleted, and gravitational energy can no longer be tapped efficiently as the degenerate electron 
pressure prevents additional contraction. Because this pressure is independent of the temperature, a white dwarf is condemned to evolve at essentially constant radius. The mechanical structure of such a star is therefore specified by the degenerate electrons. In particular, electron degeneracy is directly responsible for the mass-radius relation of white dwarf stars, as well as for the limiting (Chandrasekhar) mass above which a white dwarf cannot exist. The latter is of the order of 1.2-1.4 $\mathrm{M}_{\odot}$.

Degenerate electrons also possess another property of high relevance for white dwarfs: they are excellent conductors of heat, and thus thermalize efficiently the internal regions of white dwarfs. We can thus envision a white dwarf as an almost isothermal core containing typically more than $99.99 \%$ of its mass and surrounded by a thin, opaque, insulating, non-degenerate outer layer. Typical internal temperatures range from $\sim 2 \times 10^{7} \mathrm{~K}$ to $\sim 5 \times 10^{6} \mathrm{~K}$ for stars in the range $16,000 \mathrm{~K}-8000 \mathrm{~K}$, the most common white dwarfs. The very large temperature drop between the central regions and the surface takes place mainly in the stellar envelope, and usually leads to the formation of convection zones which play a key role in the evolution of the white dwarf by affecting directly the cooling rate.

Largely decoupled from the electrons, the non-degenerate ions provide the thermal energy which slowly leaks through the outside, thereby producing the star's Juminosity. The electrons do not contribute to the energy reservoir because degenerate particles cannot be cooled. As thermal energy is gradually lost from the star in the form of radiation, the ion system evolves from a gas to a fluid to a solid. The cooling of white dwarfs is thus accompanied by a small and gradual change in the physical conditions of the internal, generally unaccessible, regions of the star. The associated timescales are long, of the order of $10^{8}-10^{9}$ yr. Amazingly enough, however, accurate observations of pulsating white dwarfs now afford us, in principle, the possibility of observing these slow, secular changes in the internal structure of degenerate stars.

\section{Watching White Dwarfs Cool}

A particularly powerful way of probing the inner regions of a white dwarf is provided by the existence of so-called instability strips along the cooling sequences of white dwarfs. For white dwarf stars with hydrogen-rich surface composition, the strip is located in the range $11,000 \mathrm{~K} \lesssim T_{\text {eff }} \lesssim 13,000 \mathrm{~K}$, and its existence is intimately connected to the presence of an extensive hydrogen convection zone in the envelope. These instabilities manifest themselves as temperature waves at the stellar surface which cause multiperiodic luminosity variations. Because the pulsation properties of a star depend on its global structure, a comparison of the observed period structure of a white dwarf with those of models provides a unique way to probe the internal structure of white dwarfs. In particular, the slow cooling of the internal regions of a pulsating white dwarf is expected to leave its mark on the light curve of that object in the form of a slow drift in the pulsation period of individual pulsation modes. The tantalizing possibility of witnessing white dwarf cooling has been recently exploited by Kepler et al. (1991), who report the first detection of the rate of change of the main pulsation period of the variable DA white dwarf G117-B15A, the crowning result of some 14 years of painstaking observations. The observed rate of change of the mode at $215 \mathrm{~s}$ in $\mathrm{G} 117-\mathrm{B} 15 \mathrm{~A}$ is $\Pi=12.0 \pm 3.5 \times 10^{-15} \mathrm{~s} \mathrm{~s}^{-1}$.

With the help of cooling and pulsation calculations, it is possible to interpret the 
observed value of $\dot{\Pi}$ in terms of the core composition of G117-B15A. In Mestel's (1952) theory of white dwarf cooling, $\dot{I}$ is simply proportional to $A$, the mean atomic weight of the core. This relationship was thoroughly investigated by Brassard et al. (1991), and their main result is summarized in Figure 1.

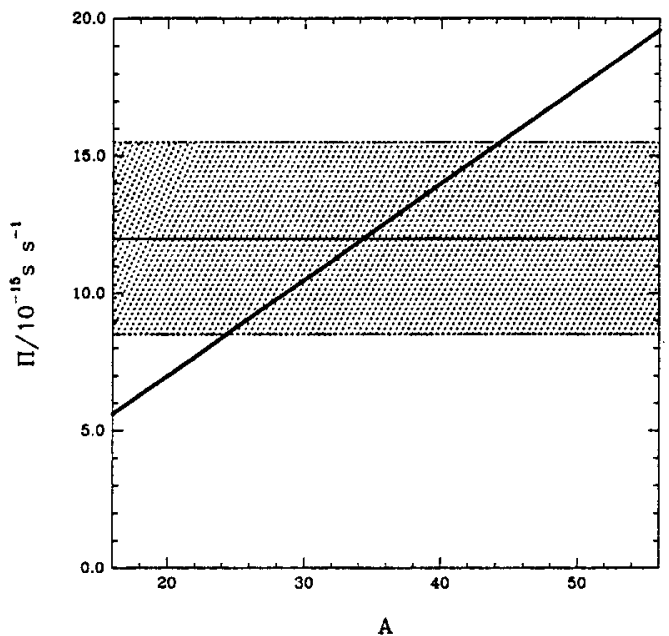

Figure 1. Predicted rate of period change (thick solid line) as a function of mean atomic weight of the core for modes near $215 \mathrm{~s}$ in models representative of G117-B15A. The observed rate of period change and the range allowed by the $1 \sigma$ limits are also indicated.

Taken at face value, the Kepler et al. result implies $A=34 \pm 10$. In other words, the core of G117-B15A appears to be made of elements at least as heavy as magnesium (at the one-sigma level). This result is quite surprising, as our canonical knowledge of white dwarfs suggests that their cores ought to be composed of carbon and oxygen instead. While the observed rate of period change remains, at the $2 \sigma$ level, consistent with the presence of a carbon core, and while other mechanisms (such as the existence of a Jupiter-like planet around G117-B15A) might be called upon to account for the large value of $\dot{I}$ measured in that star (see Brassard et al. 1991), the possibility remains that our understanding of pre-white dwarf evolution and of white dwarf cooling might be incomplete. With its potential barely tapped, asteroseismology will remain the best tool at our disposal to probe directly the inner structure, and thermal evolution, of white dwarf stars.

This work was supported in part by the NSERC Canada and by the Fund FCAR (Québec). G. Fontaine also acknowledges financial support from a Killam Fellowship.

\section{REFERENCES}

Brassard, P., Fontaine, G., Wesemael, F., Kepler, S.O., Wood, M.A., Bergeron, P., and McGraw, J.T. 1991, Ap.J., in press.

Kepler, S.O., et al. 1991, Ap.J. (Letters), in press.

Mestel, L. 1952, M.N.R.A.S., 112, 583. 